\title{
High-Rate Codes With Bounded PMEPR for BPSK and Other Symmetric Constellations
}

\author{
Masoud Sharif, Member, IEEE, and Babak Hassibi
}

\begin{abstract}
In this letter, we consider the problem of constructing high-rate codes with low peak-to-mean-envelope power ratio (PMEPR) for multicarrier signals. Assuming coefficients of the multicarrier signal are chosen from a symmetric $q$-ary constellation, we construct codes with rate $1-(1 / r) \log _{q} 2$ and PMEPR of less than $c r \log n$ for any $r$ and $n$, where $n$ is the number of subcarriers and $c$ is a constant independent of $n$ and $r$. The construction is based on dividing $n$ subcarriers into $n / r$ groups of $r$ subcarriers and choosing a sign for each group to minimize the PMEPR. The signs are chosen using a variation of the algorithm proposed by the authors in previous papers. For large $n$, we can, in fact, construct a code with a rate of $1-O(1 / \log n)$ and PMEPR of less than $c \log ^{2} n$. For binary phase-shift-keying-modulated signals, this partially solves the problem posed by Litsyn and implies a construction of $2^{n / 2}$ codewords with PMEPR less than $2 c \log n$.
\end{abstract}

\section{INTRODUCTION}

$\mathbf{M}$ ULTICARRIER signals are proposed in many high-speed wireless and wireline standards such as WLAN 802.11, xDSL, and DVB. In a typical multicarrier signal, the transmitted signal consists of a large number of subcarriers (e.g., 64 for the WLAN standard) that leads to a signal with high peak-to-mean-envelope-power ratio (PMEPR). This large PMEPR requires a highly linear power amplifier in the transmitter front end that consequently hampers its power efficiency.

Over the years, a whole host of methods have been developed for PMEPR reduction such as coding, deliberate clipping, selective mapping (SLM), reserved carriers, and tone injection [4]-[10]. Of course, PMEPR reduction comes at a price in terms of coding rate, average power, signal distortion, and bandwidth. Methods like coding usually give a worst-case guarantee on the PMEPR. On the other hand, there are other methods, such as SLM and partial transmit sequence (PTS), that improve the probability distribution of the PMEPR, i.e., reduce the probability of encountering a large PMEPR. In this letter, we propose a coding scheme that reduces the PMEPR at the expense of a rate hit.

Paper approved by Y. Li, the Editor for Wireless Communications Theory of the IEEE Communications Society. Manuscript received May 5, 2004; revised January 5, 2005. This work was supported in part by the National Science Foundation under Grant CCR-0133818, in part by the Office of Naval Research under Grant N00014-02-1-0578, and in part by Caltech's Lee Center for Advanced Networking.

M. Sharif was with the Department of Electrical Engineering, California Institute of Technology, Pasadena, CA 91125 USA. He is now with the Department of Electrical and Computer Engineering, Boston University, Boston, MA 02215 USA (e-mail: sharif@bu.edu).

B. Hassibi is with the Department of Electrical Engineering, California Institute of Technology, Pasadena, CA 91125 USA (e-mail: hassibi@ systems.caltech.edu).

Digital Object Identifier 10.1109/TCOMM.2006.877964
Recently, in [1], an algorithm was proposed to choose the sign of each subcarrier in order to reduce the PMEPR. In other words, for a symmetric $q$-ary constellation, a code is constructed with rate $1-\log _{q} 2$ and PMEPR of less than $c \log n$ for any $n$, where $c$ is a constant independent of $n$. The algorithm works only for constellations larger than binary phase-shift keying (BPSK), i.e., $q>2$. For BPSK constellations, recently in [10], the authors characterized the codewords with PMEPR higher than $(3 / 4) n$. This motivates the question of whether we can construct exponentially many codewords from BPSK with PMEPR bounded by $\log n$ [3].

In this letter, we partially solve the aforementioned problem. We propose an algorithm that only uses the signs of $(n / r)$ subcarriers and guarantees that the PMEPR of the resulting codeword is less than $c r \log n$ for any $n$ and any $r .{ }^{1}$ For a BPSK constellation, this implies a code with rate $1-(1 / r)$ and PMEPR less than $c r \log n$. In general, for a symmetric $q$-ary constellation, we construct a code with rate $1-(1 / r) \log _{q} 2$ and a PMEPR of less than $c r \log n$. It is also worth noting that all the complexity in the encoding is to find the optimum signs, which can be done in polynomial time, and the decoding is also simple, as the transmitter does not send any information over the signs of $(n / r)$ subcarriers. An interesting consequence of this result is the construction of codes with rate $1-O(1 / \log n)$ (almost full rate) and PMEPR of less than $c \log ^{2} n$. Simulation results show a significant PMEPR reduction with a little rate loss.

The letter is organized as follows. Section II introduces our notation and the statement of the problem. In Section III, the main result of the letter is presented, and high-rate codes with bounded PMEPR are constructed. Section IV discusses extensions of our work and open problems. Simulation results are presented in Section V, and Section VI concludes the paper.

\section{Definitions and Problem Statement}

We may write the normalized multicarrier signal $s_{C}(\theta)$ that consists of $n$ subcarriers as

$$
s_{C}(\theta)=\sum_{i=1}^{n} c_{i} e^{j \theta i}
$$

where $C=\left(c_{1}, \ldots, c_{n}\right)$ is the modulating vector (or the codeword), $c_{i}$ 's are chosen from a constellation such as BPSK or quadrature amplitude modulation (QAM), and $\theta$ denotes time. Clearly, if the $c_{i}$ 's are chosen from an $M$-ary (M)PSK constellation and they add up coherently, $s_{C}(\theta)$ will have a large peak of amplitude $n$.

\footnotetext{
${ }^{1}$ Throughout the letter, $c$ is a constant independent of $n$ and $r$.
} 
In order to evaluate the variation of the amplitude of a multicarrier signal, we may define the PMEPR of $C$ as

$$
\operatorname{PMEPR}(C)=\max _{0 \leq \theta \leq 2 \pi} \frac{\left|s_{C}(\theta)\right|^{2}}{E\left\{\sum_{i=1}^{n}\left|c_{i}\right|^{2}\right\}}
$$

where the denominator is the average power of $s_{C}(\theta)$. Thus, if $c_{i}$ 's are chosen independently from a constellation with average power of $P_{\mathrm{av}}$, then $E\left\{\sum_{i=1}^{n}\left|c_{i}\right|^{2}\right\}=n P_{\mathrm{av}}$.

In this letter, we consider PMEPR reduction by adjusting the sign of some of the subcarriers. This method is a more general version of the scheme that has been recently proposed in [1], and it is shown that by adjusting the signs of all subcarriers, we can achieve constant PMEPR with a little rate hit for large $n$ [11]. In [1] and [2], an algorithm is also proposed to design the signs such that the resulting PMEPR is guaranteed to be less than $c \log n$ for any $n$.

Therefore, by using the sign of each subcarrier to reduce the PMEPR, we can construct a code with $(q / 2)^{n}$ codewords (out of $q^{n}$ ) that has a PMEPR of less than $c \log n$ [2]. To quantify this reduction in the number of codewords, we may define the rate of a code $\mathcal{C}$ as

$$
R=\frac{1}{n} \log _{q}|\mathcal{C}|
$$

where $c_{i}$ 's are chosen from a $q$-ary constellation and $|\mathcal{C}|$ denotes the cardinality of the set $\mathcal{C}$. Clearly, based on our definition of rate, "full-rate codes" refers to $R=1$ for any $q$.

It has been shown that almost all the $q^{n}$ codewords have a PMEPR of less than $\log n+O(\log \log n)$ for various kinds of constellations, including symmetric ones, ${ }^{2}$ when $n$ is large [12]-[14]. However, there is no explicit construction of an almost rate-one code that approaches that limit for PMEPR. For BPSK, there is even no construction of exponentially many codewords with PMEPR less than $c \log n$ [3].

In this letter, we address both of the aforementioned problems. Namely, we construct codes that have almost full rate, i.e., $[R=1-O(1 / \log n)]$ and a PMEPR of less than $c \log ^{2} n$ for large $n$. Furthermore, for BPSK constellations, we propose codes with rate $R=1-1 / r$ and PMEPR less than $c r \log n$.

\section{High-Rate Codes With Bounded PMEPR}

Unlike [2], where the signs of all subcarriers are used to reduce the PMEPR, we consider the problem of using a fraction of all the signs to reduce the PMEPR. This is, in fact, motivated by the fact that for BPSK, there is no construction for a high-rate code with PMEPR of less than $O(\log n)$ (see [10] and [3]) and the scheme of [2] does not work for BPSK, as it uses the signs of all subcarriers to reduce the PMEPR.

In order to construct BPSK codewords with bounded PMEPR, for any integer $r$, we use the sign of $(n / r)$ equally spaced subcarriers indexed from 1 to $n$ to minimize the

\footnotetext{
${ }^{2}$ By symmetric constellation, here we mean that if a point $A$ belongs to the
} constellation, $-A$ will also be in the constellation.
PMEPR. ${ }^{3}$ Therefore, for any given codeword $C$, we would like to solve the following nonconvex problem:

$$
\min _{\epsilon_{1}, \epsilon_{2}, \ldots} \max _{0 \leq \theta \leq 2 \pi}\left|\sum_{i=0}^{\frac{n}{r}-1} \epsilon_{i+1}\left(\sum_{s=1}^{r} c_{i r+s} e^{j \theta(i r+s)}\right)\right|
$$

where $\epsilon_{i} \in\{+1,-1\}$. It is worth mentioning that setting $r=1$ reduces the problem to the one addressed in [2].

Following [2], instead of maximizing over $0 \leq \theta \leq 2 \pi$, we can minimize the maximum over $k n$ samples of $\theta$ [15], [16]. Therefore, the min-max problem of (4) can be written as

$$
\min _{\epsilon_{1}, \epsilon_{2}, \ldots} \max _{1 \leq p \leq 2 k n}\left|\sum_{i=0}^{\frac{n}{r}-1} \epsilon_{i+1} a_{p i}\right|
$$

where

$$
a_{p i}= \begin{cases}\Re\left\{\sum_{s=1}^{r} c_{i r+s} e^{j \theta(i r+s)}\right\}, & 1 \leq p \leq k n \\ \Im\left\{\sum_{s=1}^{r} c_{i r+s} e^{j \theta(i r+s)}\right\}, & k n+1 \leq p \leq 2 k n\end{cases}
$$

and $\theta_{p}=2 \pi p /(k n)$.

Fortunately, the machinery used in [2] can be generalized to this case, and the following algorithm can be deduced by using a derandomization method, as we did in [2].

Algorithm 1: For any $C=\left(c_{1}, \ldots, c_{n}\right)$, let $k$ be an integer greater than 1 and $\left|c_{i}\right| \leq \sqrt{E_{\max }}$. Then $\epsilon_{1}=1$, and $\epsilon_{s}$ 's are recursively determined as

$$
\begin{aligned}
\epsilon_{j}=-\operatorname{sign}\left\{\sum_{p=1}^{2 k n} \sinh \left\{\alpha^{*} \sum_{r=1}^{s-1} \epsilon_{r} a_{\mathrm{pr}}\right\}\right. & \\
& \left.\times \sinh \left(\alpha^{*} a_{\mathrm{ps}}\right) \prod_{r=s+1}^{n / r} \cosh \left\{\alpha^{*} a_{\mathrm{pr}}\right\}\right\}
\end{aligned}
$$

for $s=2, \ldots, n / r$, where $\alpha^{*}=\sqrt{2 \log 4 k n /\left(n r E_{\max }\right)}$.

The next theorem gives a guarantee on the PMEPR of the resulting codeword by using Algorithm 1 .

Theorem 1: Let $C$ be a given codeword where $c_{i} \leq$ $\sqrt{E_{\max }}$ and $E_{\mathrm{av}}=E\left\{\left|c_{i}\right|^{2}\right\}$. Also let $C_{\epsilon}=\left(\epsilon_{1} c_{1}, \ldots\right.$, $\left.\epsilon_{1} c_{r-1}, \epsilon_{2} c_{r}, \ldots\right)$ where $\epsilon_{i}$ 's are determined according to Algorithm 1. Then the PMEPR of $C_{\epsilon}$ is less than $4 r E_{\max } /\left(\cos ^{2}(\pi / 2 k) E_{\mathrm{av}}\right) \log 4 k n$ for any $n$ and $r$ where $k$ is a positive integer such that $k n$ is an integer.

Proof: The proof is along the same line as the proof of $[2$, Th. 3]. The only difference here is that we are minimizing the maximum of $2 k n$ linear forms over $n / r$ signs, as opposed to $n$ in [2].

Remark 1: It is worth mentioning that our scheme is similar to the PTS method in that we search for the optimum sign for each group to minimize the PMEPR. The difference, however, is that we do not require side information in the receiver, as the signs used for PMEPR reduction do not carry any information. Moreover, we propose a simple deterministic algorithm that provides a guarantee on the PMEPR without performing any Fourier transformation [8].

Remark 2: Since $k$ is a constant, Theorem 1 implies that the resulting codeword has a PMEPR of less than $c \log n$, where $c$ is

\footnotetext{
${ }^{3}$ For simplicity, we always assume $r$ divides $n$. This condition is not necessary, and it is just for simplifying the notations.
} 
a constant independent of $n$ and $r$, and that $c$ can be determined by optimizing over $k$.

We can now construct a code set $\mathcal{C}$ such that the PMEPR of all its codewords is less than $c r \log n$ when the $c_{i}$ 's are chosen from a symmetric $q$-ary constellation. This can be done by reserving the sign of only $n / r$ subcarriers (indexed $i=1, r+1,2 r+$ $1, \ldots, n-r+1)$ to minimize the PMEPR over those signs. Given all the $c_{i}$ 's, Algorithm 1 can be used to determine the signs in polynomial time. Therefore, we end up having $(q / 2)^{n / r} q^{n-n / r}$ codewords with a PMEPR of less than $c r \log n$ for any $n$ and $r$. That leads to the following corollary.

Corollary 1: If $c_{i}$ 's are chosen from a $q$-ary constellation, the code $\mathcal{C}$ constructed using Algorithm 1 has a rate of $1-$ $(1 / r) \log _{q} 2$, and its PMEPR is less than $c r \log n$ for any $n$ and $r$, where $c$ is a constant independent of $n$ and $r$.

It is worth noting that the decoding of $\mathcal{C}$ is quite simple, as the decoder can infer the signs $\left(\epsilon_{i}\right.$ 's) from the corresponding subcarriers, since they do not convey any information over their signs.

Remark 3: The extension of our algorithm to the case where $\epsilon_{i}$ 's can be chosen from $\{ \pm 1 \pm j\}$ is straightforward. In this case, $\epsilon_{i}$ 's in (4) should be replaced by $\epsilon_{i}+j \epsilon_{i}^{\prime}$, where $\epsilon_{i}$ and $\epsilon_{i}^{\prime}$ are chosen from $\{+1,-1\}$. Therefore, using the same argument as in (5), the problem can be again written in a similar form as in (5) and can be solved using Algorithm 1.

In the next two subsections, we further look into two special cases, namely, we look into codes for BPSK constellations, and also the PMEPR-rate region of the codes when $n$ is large.

\section{A. Example: BPSK Constellations}

The famous result of Halasz [12] states that almost all BPSK codewords have a PMEPR of less than $\log n+O(\log \log n)$ for large $n$. The design of such a code has been recently addressed in [10], where codewords with a PMEPR of less than $(3 / 4) n$ have been characterized for any $n$.

Corollary 1 , in fact, constructs $2^{n(1-1 / r)}$ codewords with PMEPR of less than $c r \log n$ for any $n$. For fixed $r$ and large $n$, this implies a construction of exponentially many codewords (in fact, $\left.2^{n(1-1 / r)}\right)$ such that their PMEPR is $O(\log n)$.

\section{B. Large Number of Subcarriers}

In [14], the result of Halasz is extended to many other constellations, including symmetric QAM, PSK constellations and spherical codes. Therefore, the existence of codes with rate approaching one and a PMEPR of less than $\log n+O(\log \log n)$ has been established, although there is no construction close to this result [3].

Since Algorithm 1 and Theorem 1 work for any $n$ and $r$, we may choose $r$ to be $\log n$. We can, therefore, use Corollary 1 to prove that we can construct a code with rate $1-O(1 / \log n)$ and a PMEPR of less than $c \log ^{2} n$. We can make a more precise argument in the following corollary.

Corollary 2: For large $n$, using the construction as in Corollary 1 , the code $\mathcal{C}$ has a rate $1-O(1 / f(n))$ and a PMEPR of less than $c f(n) \log n$, where $1 \leq f(n) \leq n$ such that $\lim _{n \rightarrow \infty} f(n)=\infty$.

Therefore, in an attempt to construct almost rate-one codes with PMEPR of $\log n$, we have been able to construct codes

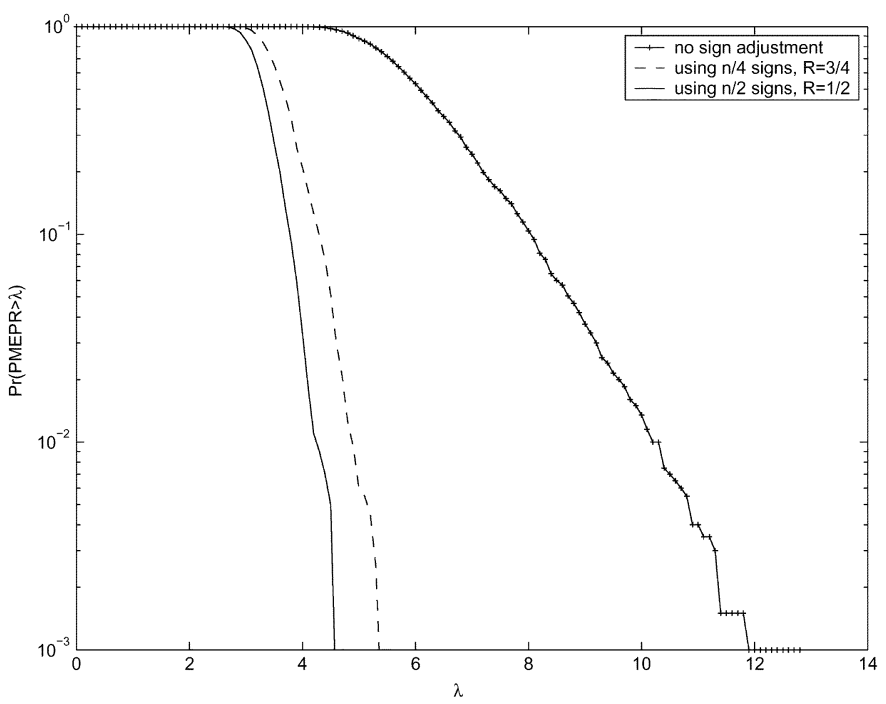

Fig. 1. CCDF of the PMEPR for BPSK by optimizing over $n / r$ signs for $r=$ 2,4 when $n=128$. This corresponds to codes with rate $1 / 2$ and $3 / 4$.

with almost rate one (in fact, $R=1-O(1 / f(n))$ ) and PMEPR of less than $c f(n) \times \log n$, where $c$ is a constant independent of $n$.

\section{EXTENSIONS AND OPEN PROBLEMS}

In [17] and [18], constellation shaping is proposed to reduce the PMEPR. In [17], it is shown that by using amplitude adjustment on top of adjusting all the signs, i.e., $r=n$, PMEPR can be further reduced at the expense of a little average power penalty. We can use the same approach, and combine our coding scheme with amplitude adjustment to further reduce the PMEPR at the expense of a lower rate hit and a little average power increase. It would be interesting to see what are the best rate and average power increase that lead to the maximum PMEPR reduction at the minimum cost.

The problem of constructing almost rate-one codes with PMEPR of $\log n+O(\log \log n)$ still remains open. Even though the result of this letter implies a construction of a code with rate almost one (i.e., $R=1-O(1 / \log n)$ ) and PMEPR of less than $c \log ^{2} n$, we are still off by a factor of $\log n$.

Another interesting problem is to investigate the performance of our algorithm (or any other algorithm) for designing the signs. Therefore, it would be useful to provide a lower bound on the value of the optimization problem posed in (5) as a benchmark. Of course, one simple lower bound can be obtained by using Parseval's theorem and bounding the maximum of the signal by its average power. However, obtaining tighter bounds remains an open problem.

Furthermore, as shown in [2], there exist nonvanishing to zero rate codes with even constant PMEPR for large $n$. Achieving codes with better PMEPR calls for a sign algorithm that has a better performance (or bound) for PMEPR. This also remains a very interesting open problem.

\section{Simulation Results}

As we discussed in the previous sections, there is a trade-off between the PMEPR reduction and the rate of a code. In this 


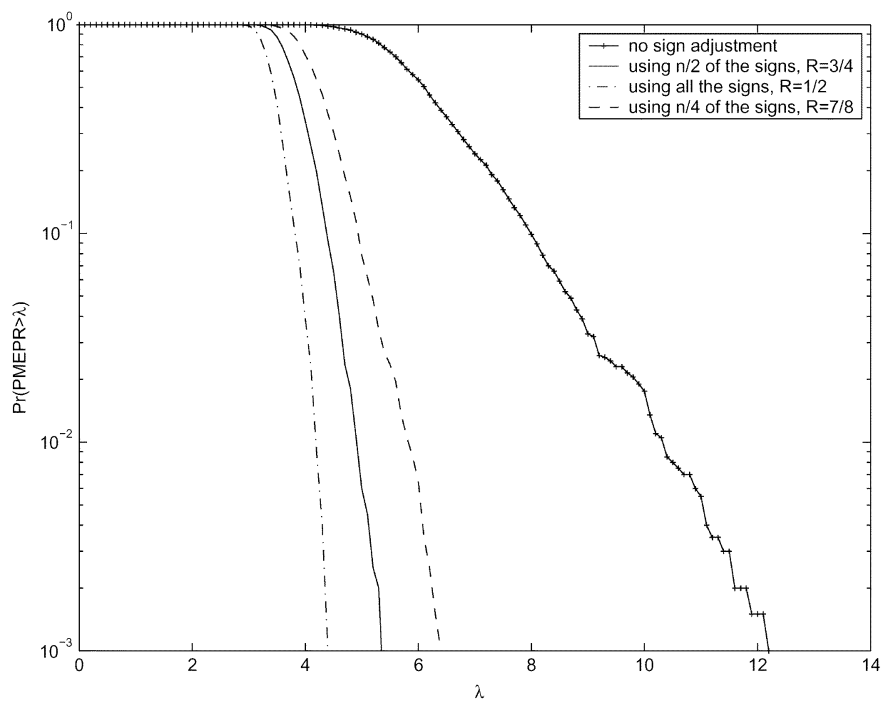

Fig. 2. CCDF of the PMEPR for QPSK by optimizing over $n / r$ signs for $r=$ $1,2,4$ when $n=128$. This corresponds to codes with rate $1 / 2,3 / 4$, and $7 / 8$.

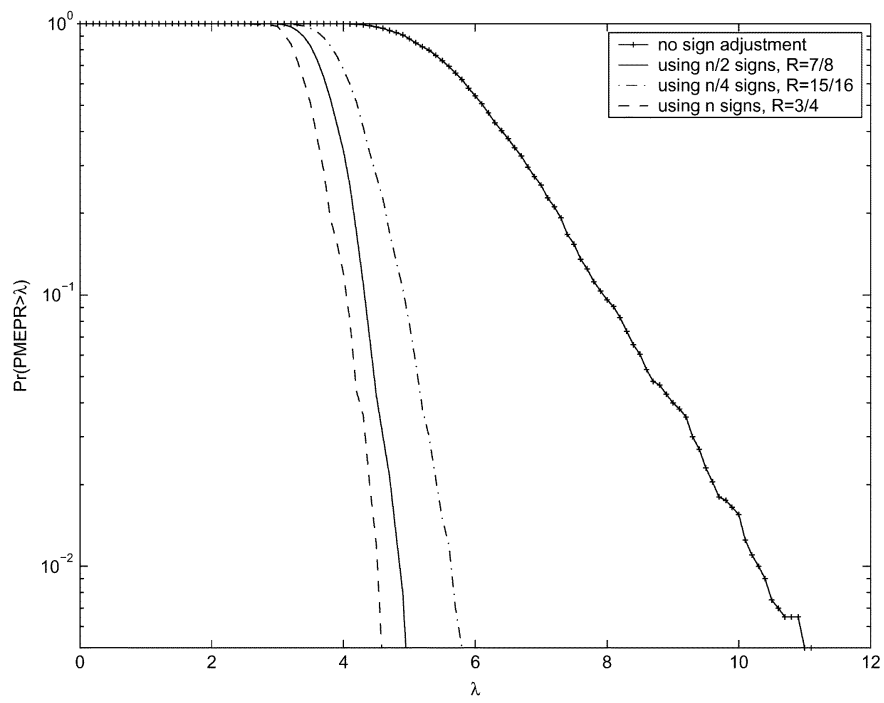

Fig. 3. CCDF of the PMEPR for 16-QAM by optimizing over $n / r$ signs for $r=1,2,4$ when $n=128$. This corresponds to codes with rate $3 / 4,7 / 8$, and $15 / 16$.

section we carry out simulations to explore this trade off for $n=128$ and for BPSK, QPSK, and 16 QAM constellations. The algorithm for designing the signs is applicable to any symmetric constellation.

Fig. 1 shows the complementary cumulative distribution function $(\mathrm{CCDF})$ of the PMEPR when $c_{i}$ 's are chosen from a BPSK constellation, and for different values of $r$ which correspond to rate $1 / 2$ and $3 / 4$ codes. As we use fewer signs (or increase the rate of code), the CCDF shifts to the right.

Figs. 2 and 3 shows the CCDF of PMEPR for quadrature (Q)PSK and 16-QAM constellations. For QPSK curves, refer to different coding rates including $1 / 2,3 / 4$, and $7 / 8$, and for 16-QAM, rates are $3 / 4,7 / 8$, and $15 / 16$. It is also worth noting that although the rate of the code highly depends on the constellation, for a fixed value of $r$, the CCDF does not really change as we change the constellation from BPSK to 16-QAM. Therefore, PMEPR reduction comes with a lower penalty in terms of rate for higher order constellations.

\section{CONCLUSION}

This letter addresses the design of high-rate codes with bounded PMEPR. By using the signs of $n / r$ subcarriers and when the $c_{i}$ 's are chosen from a $q$-ary symmetric constellation, we constructed codes with rate $1-(1 / r) \log _{q} 2$ and PMEPR of less than $c r \log n$ for any $n$ and $r$. For BPSK constellations, this implies codes with exponentially many codewords (in fact, $\left.2^{n(1-1 / r)}\right)$ that have a PMEPR of less than $c r \log n$, where $c$ is a constant independent of $n$ and $r$. For large $n$, our result implies a construction for a code with almost full rate, i.e., $1-O(1 / \log n)$, and a PMEPR of $c \log ^{2} n$. It is also worth noting that the encoding can be done in polynomial time, and the decoding is quite simple.

\section{REFERENCES}

[1] M. Sharif and B. Hassibi, "A deterministic algorithm that achieves PMEPR of $c \log n$ for multicarrier signals," in Proc. IEEE Int. Conf. Acoust., Speech, Signal Process., 2003, pp. 540-543.

[2] M. Sharif and B. Hassibi, "Existence of codes with constant PMEPR and related designs," IEEE Trans. Signal Process., vol. 52, no. 10, pp. 2836-2846, Oct. 2004.

[3] S. Litsyn, "On the PAPR problem," in DIMACS Workshop Algebraic Coding Theory, Inf. Theory, Dec. 2003, CD-ROM.

[4] K .G. Paterson and V. Tarokh, "On the existence and construction of good codes with low peak to average power ratios," IEEE Trans. Inf. Theory, vol. 46, no. 6, pp. 1974-1986, Sep. 2000.

[5] K .G. Paterson, "Generalized Reed-Muller codes and power control in OFDM modulation," IEEE Trans. Inf. Theory, vol. 46, no. 1, pp. 104-120, Jan. 2000.

[6] H. Ochiai and H. Imai, "Performance of the deliberate clipping with adaptive symbol selection for strictly bandlimited OFDM systems," IEEE J. Sel. Areas Commun., vol. 18, no. 11, pp. 2270-2277, Nov. 2000.

[7] X. Li and L. J. Cimini, "Effects of clipping and filtering on the performance of OFDM," in Proc. IEEE Veh. Technol. Conf., May 1997, pp. $1634-1638$

[8] S. H. Muller and J. B. Huber, "A comparison of peak power reduction schemes for OFDM," in Proc. IEEE Global Commun. Conf., 1997, pp. $1-5$.

[9] J. Tellado, Multicarrier Modulation with Low PAR: Applications to DSL and Wireless. Norwell, MA: Kluwer, 2000.

[10] G. Freiman, S. Litsyn, and A. Yudin, "A method to suppress high peaks in BPSK-modulated OFDM signal," IEEE Trans. Commun., vol. 52, no. 9, pp. 1440-1443, Sep. 2004.

[11] M. Sharif and B. Hassibi, "On the existence of codes with constant peak to mean envelope power ratio," in Proc. IEEE Int. Symp. Inf. Theory, 2003, p. 130.

[12] G. Halasz, "On the result of Salem and Zygmund concerning random polynomials," Studia Sci. Math. Hung., pp. 369-377, 1973.

[13] G. Wunder and H. Boche, "Upper bounds on the statistical distribution of the crest factor in OFDM transmission," IEEE Trans. Inf. Theory, vol. 49, no. 2, pp. 488-494, Feb. 2003.

[14] M. Sharif and B. Hassibi, "On multicarrier signals where the PMEPR of a random codeword is asymptotically $\log n$, , IEEE Trans. Inf. Theory, vol. 50, no. 5, pp. 895-903, May 2004.

[15] G. Wunder and H. Boche, "Peak value estimation of bandlimited signals from their samples, noise enhancement, and a local characterization in the neighborhood of an extermum," IEEE Trans. Signal Process., vol. 51, no. 3, pp. 771-780, Mar. 2003.

[16] H. Ehlich and K. Zeller, "Schwankung von polynomen zwischen gitterpunkten," (in German) Math. Zeitschr., vol. 86, pp. 41-44, 1964.

[17] M. Sharif, C. Florens, M. Fazel, and B. Hassibi, "Peak to average power reduction using amplitude and sign adjustment," in Proc. IEEE Int. Conf. Commun., 2004, pp. 837-841.

[18] D. Jones, "Peak power reduction in OFDM and DMT via active channel modification," in Proc. 32nd Asilomar Conf., Nov. 1999, pp. 1076-1079. 